\title{
On the behavior of solutions of the system of rational difference equations
}

$x_{n+1}=\frac{x_{n-1}}{y_{n} x_{n-1}-1}, \quad y_{n+1}=\frac{y_{n-1}}{x_{n} y_{n-1}-1}, \quad z_{n+1}=\frac{1}{y_{n} z_{n}}$

Abdullah Selçuk Kurbanli

Correspondence: akurbanli@yahoo. com

Department Of Mathematics, Faculty Of Education, Selcuk University, Konya 42090, Turkey

\section{Abstract}

In this article, we investigate the solutions of the system of difference equations $y_{n+1}=\frac{y_{n-1}}{x_{n} y_{n-1}-1}, y_{n+1}=\frac{y_{n-1}}{x_{n} y_{n-1}-1}, z_{n+1}=\frac{1}{y_{n} z_{n}}$ where $x_{0}, x_{-1}, y_{0}, y_{-1}, z_{0}, z_{-1}$ real numbers such that $y_{0} x_{-1} \neq 1, x_{0} y_{-1} \neq 1$ and $y_{0} z_{0} \neq 0$.

\section{Introduction}

In [1], Kurbanli et al. studied the behavior of positive solutions of the system of rational difference equations

$$
x_{n+1}=\frac{x_{n-1}}{y_{n} x_{n-1}+1}, y_{n+1}=\frac{y_{n-1}}{x_{n} y_{n-1}+1} .
$$

In [2], Cinar studied the solutions of the systems of difference equations

$$
x_{n+1}=\frac{1}{y_{n}}, y_{n+1}=\frac{y_{n}}{x_{n-1} y_{n-1}} \text {. }
$$

In [3], Kurbanli, studied the behavior of solutions of the system of rational difference equations

$$
x_{n+1}=\frac{x_{n-1}}{y_{n} x_{n-1}-1}, y_{n+1}=\frac{y_{n-1}}{x_{n} y_{n-1}-1}, z_{n+1}=\frac{z_{n-1}}{y_{n} z_{n-1}-1} .
$$

In [4], Papaschinnopoulos and Schinas proved the boundedness, persistence, the oscillatory behavior, and the asymptotic behavior of the positive solutions of the system of difference equations

$$
x_{n+1}=\sum_{i=0}^{k} A_{i} / y_{n-i}^{p_{i}}, \quad y_{n+1}=\sum_{i=0}^{k} B_{i} / x_{n-i}^{q_{i}}
$$

In [5], Clark and Kulenović investigate the global stability properties and asymptotic behavior of solutions of the system of difference equations

$$
x_{n+1}=\frac{x_{n}}{a+c y_{n}}, \quad y_{n+1}=\frac{y_{n}}{b+d x_{n}} .
$$

In [6], Camouzis and Papaschinnopoulos studied the global asymptotic behavior of positive solutions of the system of rational difference equations 


$$
x_{n+1}=1+\frac{x_{n}}{y_{n-m}}, y_{n+1}=1+\frac{y_{n}}{x_{n-m}}
$$

In [7], Kulenović and Nurkanović studied the global asymptotic behavior of solutions of the system of difference equations

$$
x_{n+1}=\frac{a+x_{n}}{b+y_{n}}, y_{n+1}=\frac{c+y_{n}}{d+z_{n}}, z_{n+1}=\frac{e+z_{n}}{f+x_{n}} .
$$

In [8], Özban studied the positive solutions of the system of rational difference equations

$$
x_{n+1}=\frac{1}{y_{n-k}}, y_{n+1}=\frac{y_{n}}{x_{n-m} y_{n-m-k}} .
$$

In [9], Zhang et al. investigated the behavior of the positive solutions of the system of the difference equations

$$
x_{n}=A+\frac{1}{y_{n-p}}, \quad y_{n}=A+\frac{y_{n-1}}{x_{n-r} y_{n-s}} .
$$

In [10], Yalcinkaya studied the global asymptotic stability of the system of difference equations

$$
z_{n+1}=\frac{t_{n} z_{n-1}+a}{t_{n}+z_{n-1}}, \quad t_{n+1}=\frac{z_{n} t_{n-1}+a}{z_{n}+t_{n-1}}
$$

In [11], Irićanin and Stević studied the positive solutions of the system of difference equations

$$
\begin{aligned}
& x_{n+1}^{(1)}=\frac{1+x_{n}^{(2)}}{x_{n-1}^{(3)}}, \quad x_{n+1}^{(2)}=\frac{1+x_{n}^{(3)}}{x_{n-1}^{(4)}}, \ldots, \quad x_{n+1}^{(k)}=\frac{1+x_{n}^{(1)}}{x_{n-1}^{(2)}} \\
& x_{n+1}^{(1)}=\frac{1+x_{n}^{(2)}+x_{n-1}^{(3)}}{x_{n-2}^{(4)}}, \quad x_{n+1}^{(2)}=\frac{1+x_{n}^{(3)}+x_{n-1}^{(4)}}{x_{n-2}^{(5)}}, \ldots, \quad x_{n+1}^{(k)}=\frac{1+x_{n}^{(1)}+x_{n-1}^{(2)}}{x_{n-2}^{(3)}}
\end{aligned}
$$

Although difference equations are very simple in form, it is extremely difficult to understand throughly the global behavior of their solutions, for example, see Refs. [12-34].

In this article, we investigate the behavior of the solutions of the difference equation system

$$
x_{n+1}=\frac{x_{n-1}}{y_{n} x_{n-1}-1}, \quad y_{n+1}=\frac{y_{n-1}}{x_{n} y_{n-1}-1}, \quad z_{n+1}=\frac{1}{y_{n} z_{n}}
$$

where $x_{0}, x_{-1}, y_{0}, y_{-1}, z_{0}, z_{-1}$ real numbers such that $y_{0} x_{-1} \neq 1, x_{0} y_{-1} \neq 1$ and $y_{0} z_{0} \neq 0$.

\section{Main results}

Theorem 1. Let $y_{0}=a, y_{-1}=b, x_{0}=c, x_{-1}=d, z_{0}=e, z_{-1}=f$ be real numbers such that $y_{0} x_{-1} \neq 1, x_{0} y_{-1} \neq 1$ and $y_{0} z_{0} \neq 0$. Let $\left\{x_{n}, y_{n}, z_{n}\right\}$ be a solution of the system (1.1). Then all solutions of (1.1) are

$$
x_{n}=\left\{\frac{d}{(a d-1)^{n}}\right\}, \quad n---\operatorname{odd} c(c b-1)^{n}, \quad n--- \text { even }
$$




$$
\begin{aligned}
& y_{n}=\left\{\frac{b}{\left.(c b-1)^{n}\right\}, n---\operatorname{odd} a(a d-1)^{n}, \quad n--- \text { even }}\right. \\
& z_{n}= \begin{cases}\frac{b^{n}-1}{a^{n} e[(a d-1)(c d-1)]^{\sum_{i=1}^{k} i}}, & n--- \text { odd } \\
\frac{\text { ane }(a d-1)^{\sum_{i=1}^{k}(i-1)}(c b-1)^{\sum_{i=1}^{k} i}}{b^{n}}, & n--- \text { even }\end{cases}
\end{aligned}
$$

Proof. For $n=0,1,2,3$, we have

$$
\begin{gathered}
x_{1}=\frac{x_{-1}}{y_{0} x_{-1}-1}=\frac{d}{a d-1}, \\
y_{1}=\frac{y_{-1}}{x_{0} y_{-1}-1}=\frac{b}{c b-1}, \\
z_{1}=\frac{1}{y_{0} z_{0}}=\frac{1}{a e} \\
x_{2}=\frac{x_{0}}{y_{1} x_{0}-1}=\frac{c}{\frac{b}{c b-1} c-1}=c(c b-1), \\
y_{2}=\frac{y_{0}}{x_{1} y_{0}-1}=\frac{a}{\frac{d}{a d-1} a-1}=a(a d-1) \\
z_{2}=\frac{1}{y_{1} z_{1}}=\frac{1}{\frac{b}{c b-1} \frac{1}{a e}}=\frac{(c b-1) a e}{b}=\frac{\frac{d}{a d-1}}{y_{2} x_{1}-1}=\frac{d}{a(a d-1) \frac{d}{a d-1}-1}=\frac{d}{(a d-1)^{2}}, \\
y_{3}=\frac{\frac{b}{c b-1}}{x_{2} y_{1}-1}=\frac{b}{c(c b-1) \frac{b}{c b-1}-1}=\frac{b}{(c b-1)^{2}} \\
\frac{1}{y_{2} z_{2}}=\frac{1}{a(a d-1) \frac{(c b-1) a e}{b}}=\frac{b}{a^{2} e(a d-1)(c b-1)}
\end{gathered}
$$

for $n=k$, assume that

$$
\begin{gathered}
x_{2 k-1}=\frac{x_{2 k-3}}{y_{2 k-2} x_{2 k-3}-1}=\frac{d}{(a d-1)^{k}}, \\
x_{2 k}=\frac{x_{2 k-2}}{y_{2 k-1} x_{2 k-2}-1}=c(c b-1)^{k}, \\
y_{2 k-1}=\frac{y_{2 k-3}}{x_{2 k-2} y_{2 k-3}-1}=\frac{b}{(c b-1)^{k}}, \\
y_{2 k}=\frac{y_{2 k-2}}{x_{2 k-1} y_{2 k-2}-1}=a(a d-1)^{k}
\end{gathered}
$$

and

$$
\begin{gathered}
z_{2 k-1}=\frac{b^{k-1}}{a^{k} e[(a d-1)(c b-1)]^{\sum_{i=1}^{k} i}} \\
z_{2 k}=\frac{a^{k} e(a d-1)^{\sum_{i=1}^{k}(i-1)}(c b-1)^{\sum_{i=1}^{k} i}}{b^{k}}
\end{gathered}
$$

are true. Then, for $n=k+1$ we will show that (1.2), (1.3), and (1.4) are true. From (1.1), we have 


$$
\begin{aligned}
& x_{2 k+1}=\frac{x_{2 k-1}}{y_{2 k} x_{2 k-1}-1}=\frac{\frac{d}{(a d-1)^{k}}}{a(a d-1)^{k} \frac{d}{(a d-1)^{k}}-1}=\frac{d}{(a d-1)^{k+1}}, \\
& y_{2 k+1}=\frac{\frac{b}{y_{2 k-1}}}{x_{2 k} y_{2 k-1}-1}=\frac{b}{c(c b-1)^{k}}=\frac{b}{(c b-1)^{k+1} \frac{b}{(c b-1)^{k}}-1} .
\end{aligned}
$$

Also, similarly from (1.1), we have

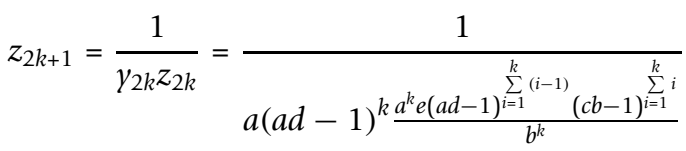

$$
\begin{aligned}
& =\frac{b^{k}}{a^{k+1} e(a d-1)^{\sum_{i=1}^{k} i}(c b-1)^{\sum_{i=1}^{k} i} .}
\end{aligned}
$$

Also, we have

$$
\begin{aligned}
& x_{2 k+2}=\frac{x_{2 k}}{y_{2 k+1} x_{2 k}-1}=\frac{c(c b-1)^{k}}{\frac{b}{(c b-1)^{k+1}} c(c b-1)^{k}-1}=\frac{c(c b-1)^{k}}{\frac{b}{(c b-1)} c-1}=c(c b-1)^{k+1}, \\
& y_{2 k+2}=\frac{y_{2 k}}{x_{2 k+1} y_{2 k}-1}=\frac{a(a d-1)^{k}}{\frac{d}{(a d-1)^{k+1}} a(a d-1)^{k}-1}=\frac{a(a d-1)^{k}}{\frac{d}{(a d-1)} a-1}=a(a d-1)^{k+1}
\end{aligned}
$$

and

$$
\begin{aligned}
& z_{2 k+2}=\frac{1}{y_{2 k+1} z_{2 k+1}}=\frac{1}{\frac{b}{(c b-1)^{k+1}} \frac{b^{k}}{a^{k+1} e(a d-1)^{i=1} i(c b-1)^{\sum_{i=1}^{k} i}}} \\
& =\frac{a^{k+1} e(a d-1)^{\sum_{i=1}^{k} i}(c b-1)^{\sum_{i=1}^{k+1} i}}{b^{k+1}}=\frac{a^{k+1} e(a d-1)^{\sum_{i=1}^{k+1}(i-1)}(c b-1)^{\sum_{i=1}^{k+1} i}}{b^{k+1}} .
\end{aligned}
$$

Corollary 1. Let $\left\{x_{n}, y_{n}, z_{n}\right\}$ be a solution of the system (1.1). Let $a, b, c, d, e, f$ be real numbers such that $a d \neq 1, c b \neq 1, a e \neq 0$ and $b \neq 0$. Also, if $a d, c b \in(1,2)$ and $b>a$ then we have

$$
\lim _{n \rightarrow \infty} x_{2 n-1}=\lim _{n \rightarrow \infty} y_{2 n-1}=\lim _{n \rightarrow \infty} z_{2 n-1}=\infty
$$

and

$$
\lim _{n \rightarrow \infty} x_{2 n}=\lim _{n \rightarrow \infty} y_{2 n}=\lim _{n \rightarrow \infty} z_{2 n}=0
$$

Proof. From $a d, c b \in(1,2)$ and $b>a$ we have $0<a d-1<1$ and $0<c b-1<1$.

Hence, we obtain

$$
\begin{array}{ll}
\lim _{n \rightarrow \infty} x_{2 n-1}=\lim _{n \rightarrow \infty} \frac{d}{(a d-1)^{n}}=d \lim _{n \rightarrow \infty} \frac{1}{(a d-1)^{n}}=d . & \infty=\left\{\begin{array}{l}
-\infty, d<0 \\
+\infty, d>0
\end{array},\right. \\
\lim _{n \rightarrow \infty} y_{2 n-1}=\lim _{n \rightarrow \infty} \frac{b}{(c b-1)^{n}}=b \lim _{n \rightarrow \infty} \frac{1}{(c b-1)^{n}}=b . & \infty= \begin{cases}-\infty, & b<0 \\
+\infty, & b>0\end{cases}
\end{array}
$$


and

$$
\lim _{n \rightarrow \infty} z_{2 n-1}=\lim _{n \rightarrow \infty} \frac{b^{n-1}}{a^{n} e[(a d-1)(c b-1)]^{\sum_{i=1}^{k} i}}=\frac{1}{e} . \quad \infty=\left\{\begin{array}{l}
-\infty, e<0 \\
+\infty, e>0
\end{array}\right.
$$

Similarly, from $a d, c b \in(1,2)$ and $b>a$, we have $0<a d-1<1$ and $0<c b-1<1$.

Hence, we obtain

$$
\begin{aligned}
& \lim _{n \rightarrow \infty} x_{2 n}=\lim _{n \rightarrow \infty} c(c d-1)^{n}=c \lim _{n \rightarrow \infty}(c d-1)^{n}=c . \quad 0=0, \\
& \lim _{n \rightarrow \infty} y_{2 n}=\lim _{n \rightarrow \infty} a(a f-1)^{n}=a \lim _{n \rightarrow \infty}(a f-1)^{n}=a . \quad 0=0 .
\end{aligned}
$$

and

$$
\lim _{n \rightarrow \infty} z_{2 n}=\lim _{n \rightarrow \infty} \frac{a^{n} e(a d-1) \sum_{i=1}^{k}(i-1)}{(c b-1) \sum^{\sum_{i=1}^{k} i}}=0 . e . \quad 0=0 .
$$

$\square$

Corollary 2. Let $\left\{x_{n}, y_{n}, z_{n}\right\}$ be a solution of the system (1.1). Let $a, b, c, d, e, f$ be real numbers such that $a d \neq 1, c b \neq 1$, ae $\neq 0$ and $b \neq 0$. If $a=b$ and $c b=a d=2$ then we have

$$
\begin{aligned}
& \lim _{n \rightarrow \infty} x_{2 n-1}=d, \\
& \lim _{n \rightarrow \infty} y_{2 n-1}=b \\
& \lim _{n \rightarrow \infty} z_{2 n-1}=\frac{1}{a e}
\end{aligned}
$$

and

$$
\begin{aligned}
& \lim _{n \rightarrow \infty} x_{2 n}=c, \\
& \lim _{n \rightarrow \infty} y_{2 n}=a, \\
& \lim _{n \rightarrow \infty} z_{2 n}=e .
\end{aligned}
$$

Proof. From $a=b$ and $c b=a d=2$ then we have, $c b-1=a d-1=1$. Hence, we have

$$
\lim _{n \rightarrow \infty}(c b-1)^{n}=1
$$

and

$$
\lim _{n \rightarrow \infty}(a d-1)^{n}=1 .
$$

Also, we have

$$
\begin{array}{ll}
\lim _{n \rightarrow \infty} x_{2 n-1}=\lim _{n \rightarrow \infty} \frac{d}{(a d-1)^{n}}=d \lim _{n \rightarrow \infty} \frac{1}{(a d-1)^{n}}=d . & 1=d, \\
\lim _{n \rightarrow \infty} y_{2 n-1}=\lim _{n \rightarrow \infty} \frac{b}{(c b-1)^{n}}=b \lim _{n \rightarrow \infty} \frac{1}{(c b-1)^{n}}=b . & 1=b
\end{array}
$$

and

$$
\lim _{n \rightarrow \infty} z_{2 n-1}=\lim _{n \rightarrow \infty} \frac{b^{n-1}}{a^{n} e[(a d-1)(c b-1)]^{\sum_{i=1}^{K} i}}=\lim _{n \rightarrow \infty} \frac{1}{a e} \frac{b^{n-1}}{a^{n-1}[(a d-1)(c b-1)]^{\sum_{i=1}^{k} i}}=\frac{1}{a e} .
$$


Similarly, we have

$$
\begin{aligned}
& \lim _{n \rightarrow \infty} x_{2 n}=\lim _{n \rightarrow \infty} c(c b-1)^{n}=c \lim _{n \rightarrow \infty}(c b-1)^{n}=c . \quad 1=c, \\
& \lim _{n \rightarrow \infty} y_{2 n}=\lim _{n \rightarrow \infty} a(a d-1)^{n}=a \lim _{n \rightarrow \infty}(a d-1)^{n}=a . \quad 1=a \text {. }
\end{aligned}
$$

and

$$
\lim _{n \rightarrow \infty} z_{2 n}=\lim _{n \rightarrow \infty} \frac{a^{n} e(a d-1)^{\sum_{i=1}^{k}(i-1)}(c b-1)^{\sum_{i=1}^{k} i}}{b^{n}}=1 \cdot e=e .
$$

Corollary 3. Let $\left\{x_{n}, y_{n}, z_{n}\right\}$ be a solution of the system (1.1). Let $a, b, c, d$, e, f be real numbers such that $a d \neq 1, c b \neq 1, a e \neq 0$ and $b \neq 0$. Also, if $0<a, b, c, d, e, f<1$ then we have

$$
\lim _{n \rightarrow \infty} x_{2 n}=\lim _{n \rightarrow \infty} y_{2 n}=\lim _{n \rightarrow \infty} z_{2 n}=0
$$

and

$$
\lim _{n \rightarrow \infty} x_{2 n-1}=\lim _{n \rightarrow \infty} y_{2 n-1}=\lim _{n \rightarrow \infty} z_{2 n-1}=\infty .
$$

Proof. From $0<a, b, c, d, e, f<1$ we have $-1<a d-1<0$ and $-1<c b-1<0$. Hence, we obtain

$$
\begin{array}{ll}
\lim _{n \rightarrow \infty} x_{2 n}=\lim _{n \rightarrow \infty} c(b c-1)^{n}=c \lim _{n \rightarrow \infty}(b c-1)^{n}=c . & 0=0, \\
\lim _{n \rightarrow \infty} y_{2 n}=\lim _{n \rightarrow \infty} a(a d-1)^{n}=a \lim _{n \rightarrow \infty}(a d-1)^{n}=a . & 0=0
\end{array}
$$

and

$$
\lim _{n \rightarrow \infty} z_{2 n}=\lim _{n \rightarrow \infty} \frac{a^{n} e(a d-1) \sum_{i=1}^{k}(i-1)(c b-1)^{\sum_{i=1}^{k} i}}{b^{n}}=e . \quad 0=0 .
$$

Similarly, we have

$$
\begin{gathered}
\lim _{n \rightarrow \infty} x_{2 n-1}=\lim _{n \rightarrow \infty} \frac{d}{(a d-1)^{n}}=d \lim _{n \rightarrow \infty} \frac{1}{(a d-1)^{n}}=d \lim _{n \rightarrow \infty} \frac{1}{(a d-1)^{n}}=d . \quad \infty=\left\{\begin{array}{l}
-\infty, n-\text { odd } \\
+\infty, n-\text { even }
\end{array},\right. \\
\lim _{n \rightarrow \infty} y_{2 n-1}=\lim _{n \rightarrow \infty} \frac{b}{(b c-1)^{n}}=b \lim _{n \rightarrow \infty} \frac{1}{(b c-1)^{n}}=b . \quad \infty=\left\{\begin{array}{l}
-\infty, n-\text { odd } \\
+\infty, n-\text { even } .
\end{array}\right.
\end{gathered}
$$

and

$$
\lim _{n \rightarrow \infty} z_{2 n-1}=\lim _{n \rightarrow \infty} \frac{b^{n-1}}{a^{n} e[(a d-1)(c b-1)]^{\sum_{i=1}^{k} i}}=+\infty .
$$

Corollary 4. Let $\left\{x_{n}, y_{n}, z_{n}\right\}$ be a solution of the system (1.1). Let $a, b, c, d, e, f$ be real numbers such that $a d \neq 1, c b \neq 1, a e \neq 0$, and $b \neq 0$. Also, if $0<a, b, c, d, e, f<1$ then we have

$$
\begin{aligned}
& \lim _{n \rightarrow \infty} x_{2 n} y_{2 n-1}=c b, \\
& \lim _{n \rightarrow \infty} x_{2 n-1} y_{2 n}=a d
\end{aligned}
$$


and

$$
\lim _{n \rightarrow \infty} z_{2 n-1} z_{2 n}=\infty
$$

\section{Proof. The proof is clear from Theorem 1. $\square$}

\section{Competing interests}

The author declares that they have no competing interests.

\section{Received: 2 March 2011 Accepted: 6 October 2011 Published: 6 October 2011}

\section{References}

1. Kurbanli, AS, Çinar, C, Yalcinkaya, I: On the behavior of positive solutions of the system of rational difference equations $y_{n+1}=\frac{y_{n-1}}{x_{n} y_{n-1}+1}, y_{n+1}=\frac{y_{n-1}}{x_{n} y_{n-1}+1}$. Math Comput Model. 53(5-6), :1261-1267 (2011). doi:10.1016/.jmcm.2010.12.009

2. Çinar, C: On the positive solutions of the difference equation system $x_{n+1}=\frac{1}{y_{n}} y_{n+1}=\frac{y_{n}}{x_{n-1} y_{n-1}}$. Appl Math Comput. 158, 303-305 (2004). doi:10.1016/j.amc.2003.08.073

3. Kurbanli, AS: On the behavior of solutions of the system of rational difference equations $x_{n+1}=\frac{x_{n-1}}{y_{n} x_{n-1}-1}$ $z_{n+1}=\frac{z_{n-1}}{y_{n} z_{n-1}-1}, z_{n+1}=\frac{z_{n-1}}{y_{n} z_{n-1}-1}$. Discrete Dynamics Natural and Society 2011, 12 (2011). Article ID 932362

4. Papaschinopoulos, G, Schinas, CJ: On the system of two difference equations. J Math Anal Appl. 273, 294-309 (2002). doi:10.1016/50022-247X(02)00223-8

5. Clark, D, Kulenović, MRS: A coupled system of rational difference equations. Comput Math Appl. 43, 849-867 (2002). doi:10.1016/S0898-1221(01)00326-1

6. Camouzis, E, Papaschinopoulos, G: Global asymptotic behavior of positive solutions on the system of rational difference equations $x_{n+1}=1+\frac{x_{n}}{y_{n-m^{\prime}}} y_{n+1}=1+\frac{y_{n}}{x_{n-m}}$. Appl Math Lett. 17, 733-737 (2004). doi:10.1016/50893-9659(04) 90113-9

7. Kulenović, MRS, Nurkanović, Z: Global behavior of a three-dimensional linear fractional system of difference equations. J Math Anal Appl. 310, 673-689 (2005)

8. Özban, AY: On the positive solutions of the system of rational difference equations $x_{n+1}=\frac{1}{y_{n-k}}$ $y_{n+1}=\frac{y_{n}}{x_{n-m} y_{n-m-k}}$. J Math Anal Appl. 323, 26-32 (2006). doi:10.1016/j.jmaa.2005.10.031

9. Zhang, Y, Yang, X, Megson, GM, Evans, DJ: On the system of rational difference equations $\boldsymbol{X}_{n}=A+\frac{1}{\gamma_{n-p^{\prime}}}$ $y_{n}=A+\frac{y_{n-1}}{x_{n-r} y_{n-s}}$. Appl Math Comput. 176, 403-408 (2006). doi:10.1016/j.amc.2005.09.039

10. Yalcinkaya, I: On the global asymptotic stability of a second-order system of difference equations. Discrete Dyn Nat Soc 2008, 12 (2008). (Article ID 860152)

11. Irićanin, B, Stević, S: Some systems of nonlinear difference equations of higher order with periodic solutions. Dyn Contin Discrete Impuls Syst Ser A Math Anal. 13, 499-507 (2006)

12. Agarwal, RP, Li, WT, Pang, PYH: Asymptotic behavior of a class of nonlinear delay difference equations. J Difference Equat Appl. 8, 719-728 (2002). doi:10.1080/1023619021000000735

13. Agarwal, RP: Difference Equations and Inequalities. Marcel Dekker, New York, 2 (2000)

14. Papaschinopoulos, G, Schinas, CJ: On a system of two nonlinear difference equations. J Math Anal Appl. 219, 415-426 (1998). doi:10.1006/jmaa.1997.5829

15. Özban, AY: On the system of rational difference equations $x_{n}=\frac{a}{y_{n-3}} y_{n}=\frac{b y_{n-3}}{x_{n-q} y_{n-q}}$. Appl Math Comput. 188, 833-837 (2007). doi:10.1016/j.amc.2006.10.034

16. Clark, D, Kulenovic, MRS, Selgrade, JF: Global asymptotic behavior of a two-dimensional difference equation modelling competition. Nonlinear Anal. 52, 1765-1776 (2003). doi:10.1016/50362-546X(02)00294-8 17. Yang, X, Liu, Y, Bai, S: On the system of high order rational difference equations $x_{n}=\frac{a}{y_{n-p^{\prime}}} y_{n}=\frac{b y_{n-p}}{x_{n-q} y_{n-q}}$. Appl
Math Comput. 171, 853-856 (2005). doi:10.1016/.amc.2005.01.092

18. Yang, X: On the system of rational difference equations $x_{n}=A+\frac{y_{n-1}}{x_{n-p} y_{n-q^{\prime}}} y_{n}=A+\frac{x_{n-1}}{x_{n-r} y_{n-s}}$.J Math Anal Appl. 307, 305-311 (2005). doi:10.1016/i.jmaa.2004.10.045

19. Zhang, Y, Yang, $X$, Evans, DJ, Zhu, C: On the nonlinear difference equation system $x_{n+1}=A+\frac{y_{n-m}}{x_{n}}$, $y_{n+1}=A+\frac{x_{n-m}}{y_{n}}$. Comput Math Appl. 53, 1561-1566 (2007). doi:10.1016/j.camwa.2006.04.030

20. Yalcinkaya, I, Cinar, C: Global asymptotic stability of two nonlinear difference equations $z_{n+1}=\frac{t_{n}+z_{n-1}}{t_{n} z_{n-1}+a^{\prime}}$ $t_{n+1}=\frac{z_{n}+t_{n-1}}{z_{n} t_{n-1}+a}$. Fasciculi Mathematici. 43, 171-180 (2010)

21. Yalcinkaya, I, Çinar, C, Simsek, D: Global asymptotic stability of a system of difference equations. Appl Anal. 87(6), :689-699 (2008). doi:10.1080/00036810802163279

22. Yalcinkaya, I, Cinar, C: On the solutions of a systems of difference equations. Int J Math Stat Autumn. 9(A11) (2011)

23. Cinar, C: On the positive solutions of the difference equation $x_{n+1}=\frac{x_{n-1}}{1+x_{n} x_{n-1}}$. Appl Math Comput. 150, 21-24 (2004). doi:10.1016/50096-3003(03)00194-2

24. Cinar, C: On the positive solutions of the difference equation $x_{n+1}=\frac{a x_{n-1}}{1+b x_{n} x_{n-1}}$. Appl Math Comput. 156, 587-590 (2004). doi:10.1016/j.amc.2003.08.010

25. Cinar, C: On the positive solutions of the difference equation $x_{n+1}=\frac{x_{n-1}}{1+a x_{n} x_{n-1}}$. Appl Math Comput. 158, 809-812 (2004). doi:10.1016/j.amc.2003.08.140

26. Cinar, C: On the periodic cycle of $x(n+1)=\frac{a_{n}+b_{n} x_{n}}{c_{n} x_{n-1}}$. Appl Math Comput. 150, 1-4 (2004). doi:10.1016/S00963003(03)00182-6 
27. Abu-Saris, R, Çinar, C, Yalcinkaya, I: On the asymptotic stability of $x_{n+1}=\frac{a+x_{n} x_{n-k}}{x_{n}+x_{n-k}}$. Comput Math Appl. 56(5), :1172-1175 (2008). doi:10.1016/j.camwa.2008.02.028

28. Çinar, C: On the difference equation $x_{n+1}=\frac{x_{n-1}}{-1+x_{n} x_{n-1}}$. Appl Math Comput. 158, 813-816 (2004). doi:10.1016/j. amc.2003.08.122

29. Çinar, C: On the solutions of the difference equation $x_{n+1}=\frac{x_{n-1}}{-1+a x_{n} x_{n-1}}$. Appl Math Comput. 158, 793-797 (2004). doi:10.1016/i.amc.2003.08.139

30. Kurbanli, AS: On the behavior of solutions of the system of rational difference equations $x_{n+1}=\frac{x_{n-1}}{y_{n-1} x_{n-1}-1}$ $y_{n+1}=\frac{y_{n-1}}{x_{n} y_{n-1}-1}$. World Appl Sci J. (2010, in press)

31. Elabbasy, EM, El-Metwally, H, Elsayed, EM: On the solutions of a class of difference equations systems. Demonstratio Mathematica. 41(1), :109-122 (2008)

32. Elsayed, EM: On the solutions of a rational system of difference equations. Fasciculi Mathematici. 45, 25-36 (2010)

33. Elsayed, EM: Dynamics of a recursive sequence of higher order. Commun Appl Nonlinear Anal. 16(2), :37-50 (2009)

34. Elsayed, EM: On the solutions of higher order rational system of recursive sequences. Mathematica Balkanica. 21(3-4), :287-296 (2008)

doi:10.1186/1687-1847-2011-40

Cite this article as: Kurbanli: On the behavior of solutions of the system of rational difference equations $x \mathrm{n}$

$+1=x n-1 y n x n-1-1, y n+1=y n-1 x n y n-1-1, z n+1=1 y n z n$. Advances in Difference Equations 2011 2011:40.

\section{Submit your manuscript to a SpringerOpen ${ }^{\circ}$} journal and benefit from:

Convenient online submission

- Rigorous peer review

- Immediate publication on acceptance

- Open access: articles freely available online

- High visibility within the field

- Retaining the copyright to your article

Submit your next manuscript at $\boldsymbol{s p r i n g e r o p e n . c o m ~}$ 